\title{
METAMEDIOS Y PERIODISMO: REVISIÓN PANORÁMICA DE LOS NUEVOS CIBERMEDIOS
}

\author{
Metamedia and journalism: A panoramic \\ review of new media
}

José-Manuel Noguera-Vivo

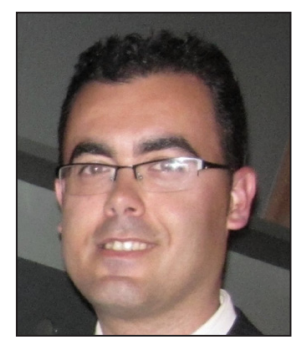

José-Manuel Noguera-Vivo es profesor titular en el área de periodismo de la Universidad Católica de Murcia (UCAM) y director del Departamento de Ciencias de la Comunicación. Dirige el grupo de investigación Comunicación, Política e Imagen y ha publicado más de una treintena de obras sobre comunicación digital, redes y periodismo. Algunos de sus libros son Todos todo. Manual de periodismo, participación y tecnología (2015), Redes y periodismo (2012) y Blogs y medios (2008). Ha sido becario postdoctoral del programa José Castillejo en University of British Columbia en Canadá (2011).

http://orcid.org/0000-0002-7189-7017

Universidad Católica de Murcia, Facultad de Comunicación Campus Los Jerónimos s/n, 30107. Guadalupe, Murcia, España jmnoguera@ucam.edu

\section{Resumen}

El presente artículo es una respuesta a un escenario mediático donde empiezan a predominar nuevos medios con características particulares, que obedecen a lo que podemos entender como "metamedios". En la medida en que estos cibermedios se sustentan en contenido ajeno o en colaboraciones con medios anteriores, el artículo plantea la necesidad de proponer una tipología que sirva para sistematizar y analizar los diferentes tipos de metamedios presentes en la Web, realizada a partir de una observación no participante. Previamente y partiendo de autores clásicos, el artículo propone una definición general de metamedio. La propuesta teórica de este trabajo supone, en esencia, ejemplos de cómo ocupar nuevos escenarios de desintermediación en el periodismo. En algunas categorías propuestas figuran plataformas como Storyful o Reported. ly, que representan características típicas de los nuevos metamedios, tanto por su manejo de contenido ajeno como por la pertinente necesidad de practicar una curación periodística de calidad.

\section{Palabras clave}

Medios; Medios digitales; Metamedios; Periodismo; Tendencias; Curación de contenido; Curación periodística; Taxonomías; Desintermediación.

\begin{abstract}
This article is a theoretical proposal for the new media landscape where many media outputs are made with third-party content. This new form of media is what we could call "metamedia", because of its singularity and how these medias are being used with other content. From this point of view, this article offers a typology (developed through non-participant observation on the internet) to analyze the new medias and by offering, at the beginning, a definition of metamedia. This article's findings point to the need to propose a taxonomy that serves to systematize and analyze the different types of metamedia present on the Web. Within this typology, some categories are especially useful to describe characteristics of these metamedias, with examples such as Storyful or Reported.ly, to understand the role of new medias as new mediators. These new medias are developing, in their daily routines, a role as "explainers" and curators of third-party content.
\end{abstract}

\section{Keywords}

Media; Digital media; Metamedia; Journalism; Trends; Content curation; Journalistic curation; Taxonomies; Disintermediation.

Noguera-Vivo, José-Manuel (2016). “Metamedios y periodismo: revisión panorámica de los nuevos cibermedios”. EI profesional de la información, v. 25, n. 3, pp. 341-350.

http://dx.doi.org/10.3145/epi.2016.may.03 


\section{Introducción}

El objetivo de este artículo es ofrecer pautas de actuación para el periodismo en un nuevo marco informativo marcado por la producción de contenido fuera de las redacciones y un consumo cada vez más determinado por el paradigma de la recomendación social. Las pautas se concretan en nuevas definiciones y clasificaciones que faciliten el estudio de los denominados metamedios.

El mencionado paradigma de recomendación causa entre otros efectos lo que hemos definido como "economía de la participación" (Noguera-Vivo et al., 2014), en la que la experiencia social alrededor del producto informativo acaba siendo parte del propio producto. Esta economía de la participación multiplica las posibilidades de asociación entre cibermedios de diferente tamaño y naturaleza. Cuanto más de nicho es un cibermedio, más posibilidades le surgen de tener una necesidad (u oportunidad) informativa que puede ser cubierta por la asociación con otro medio vertical (especializado).

Este trabajo ofrece en primer lugar claves para desarrollar el escaso marco conceptual que existe en la actualidad alrededor del concepto de metamedio (Campos-Freire, 2015).

En segundo término se describen en forma de propuesta taxonómica aquellos tipos de metamedios que mejor representan uno de los retos clave para los medios que aspiran a implementar modelos de negocio estables: ocupar nuevos escenarios de desintermediación.

Como ocurre desde hace tiempo en otros sectores con grandes gigantes de internet (Google, Facebook, eBay, Amazon...), una correcta gestión del contenido de terceros puede convertir ese contenido en un producto propio. Paradójicamente en una industria que se dedica al contenido como es el periodismo, la gestión del contenido ajeno todavía no ha terminado de desarrollarse como una vía estable de negocio y de definición de proyectos editoriales. Con la tipología que se presenta en este artículo, se pretende mostrar con claridad cómo los cibermedios también se pueden diseñar a partir de redes entre pequeños medios verticales ya existentes.

En la "economía de la participación" la experiencia social alrededor del producto informativo acaba siendo parte del propio producto

Con este trabajo se plantea la hipótesis de un escenario digital de nuevos medios donde la mayoría de ellos pueden ser descritos como metamedios por una o varias razones. Desde la acepción más tradicional del término (medios formados por medios más pequeños) hasta todo tipo de sinergias periodísticas, los nuevos cibermedios demuestran que las fórmulas de asociación y de producción en el entorno digital son numerosas. La taxonomía de metamedios propuesta es una herramienta conceptual para hacer más visible este fenómeno.
El artículo busca los siguientes objetivos alineados con esta hipótesis:

a) aportar un marco conceptual apropiado para el estudio de los nuevos metamedios;

b) proponer una tipología básica de los metamedios digitales que están caracterizando gran parte del escenario mediático actual.

\section{Una correcta gestión del contenido de terceros puede convertir ese contenido en un producto propio}

\section{Marco conceptual y definición de metamedio}

En un escenario comunicativo de máxima desintermediación como el actual, con menor importancia de los intermediarios tradicionales y creciente relevancia de otros nuevos (Facebook, Twitter, YouTube...), el periodismo todavía no se encuentra cómodo diseñando cibermedios que convivan con una realidad que ya están afrontando muchos sectores: la participación convertida en una mercancía o commodity (pero que mantiene un valor), es decir, la participación como el producto en sí mismo. Conviene aclarar que no se trata de la participación puntual paradigmática del periodismo ciudadano o del prosumer, sino de la experiencia social constante y diaria que podemos asociar a cada producto informativo (valoraciones, debates, enlaces, comentarios, RTs, compartir...).

El prosumidor acuñado originalmente por Toffler (1980) no es tal en los medios de comunicación porque tenga una constante y periódica producción de contenidos -ya que la realidad nos muestra que no es así-, sino por su capacidad potencial para producir como quiera y en cualquier momento, bajo infinidad de plataformas. El intercambio de roles emisor-receptor es continuo. En resumen:

"Todos pueden ser todo. Y aunque no lo fueran nunca en un sentido práctico, esa ilusión de participación cambia igualmente y de forma radical el escenario de medios" (Noguera-Vivo, 2015, p. 12).

La participación ciudadana ha tenido su mayor eco -en dura pugna con el periodismo ciudadano- en lo que desde la esfera científica se ha denominado "contenido generado por el usuario" o user-generated content (Van-Dijk, 2009). Pero no ha sido hasta hace unos pocos años cuando la mirada se ha vuelto hacia el punto de vista de los intereses de los medios de comunicación. Desde ahí se señala que lo rentable y estratégico ya no es tanto potenciar la producción ciudadana -puntual, imprevisible y para hechos muy ocasionales-. La estrategia ahora pasa por potenciar la circulación del contenido, lo que favorece la construcción de comunidades alrededor del cibermedio. Se teoriza sobre el denominado "contenido distribuido por usuario" o user-distributed content, y sobre la importancia de que este contenido sea objeto de una correcta curación de contenido (Villi, 2012).

El concepto de metamedio, a pesar de ser muy apropiado para definir el escenario comunicativo actual, tiene un 
breve recorrido por la literatura científica en ciencias de la comunicación. En la línea del presente artículo se pueden encontrar reflexiones acerca de la innovación disruptiva que los metamedios sociales causan en los medios tradicionales, principalmente en su modelo de negocio y cadena de valor (Campos-Freire, 2015). Sin embargo es complicado encontrar estudios que traten el impacto que supone la aparición y generalización de cibermedios de este tipo. Desde una visión muy global parece indispensable la referencia a Marshall McLuhan para recordar su premisa determinista, donde una de las bases era que los medios del futuro son construcciones holísticas de los medios actuales.

Un metamedio periodístico es un medio (o suma de medios) derivado de una interconexión novedosa de personas y contenidos preexistentes, que les sirven para definir una identidad editorial propia

$Y$ al igual que estas combinaciones de elementos conocidos generan medios desconocidos hasta la fecha, dicha aparición conlleva la obligación de normalizar nuevas prácticas -como la curación periodística-. No tendría sentido, como el propio McLuhan predecía, que ante nuevos medios no surgieran necesidades profesionales diferentes:

"En el nombre del progreso nuestra cultura oficial fuerza a los nuevos medios a hacer el trabajo de los viejos" (McLuhan; Fiore, 1967, p. 81).

Tras la propuesta genérica de McLuhan, que en realidad implicaría aceptar que todos los medios son metamedios, Kay y Goldberg (1977) fijaron su atención en el ordenador para calificarlo como el verdadero metamedio. Parecía lógico pensar que la interfaz del ordenador, con su suma de códigos, lenguajes y formatos, era la representación más literal de lo que podemos entender como un meta-medio, un medio contenedor de muchos más dentro de sí. Bajo la misma lógica, internet puede considerarse desde una triple perspectiva como metamedio:

- es el destino del resto de medios;

- es el equipo de consumo de cualquier medio;

- ese mismo equipo de consumo sirve como equipo de producción para ser un medio (Shirky, 2009).

Al margen de las primeras referencias citadas, el imaginario colectivo actual asociado al concepto de metamedio está ligado a todo tipo de redes sociales y a cualquier plataforma que gestione contenido de terceros. En suma, a todo aquello que el periodista Jean F. Fogel calificó como la "industria de flujos" en el XV Congreso de periodismo digital, de Huesca.

Para no caer en el determinismo de McLuhan y no aceptar cualquier medio contemporáneo como metamedio, creemos que cabría distinguir entre metamedios generales y periodísticos. Para ello proponemos a continuación las siguientes definiciones que ayudarán a operativizar la tipología presentada más adelante.

Entendemos por metamedio aquel medio que se apoya en estructuras, procesos, lenguajes y contenidos de medios an- teriores para crear sus propios rasgos identitarios, lenguajes, productos y hábitos de consumo. Bajo esta definición, claramente influenciada por teorías como la mediamorfosis de Fidler (1998), podemos visualizar claramente por qué internet es considerado un metamedio.

Por otra parte entendemos por metamedio periodístico aquel medio (o suma de medios) derivado de una interconexión novedosa de personas y contenidos preexistentes, los cuales les han servido para definir una identidad editorial propia y alcanzar a comunidades con nuevas necesidades informativas. Los metamedios periodísticos tienen un marcado carácter holístico, ya que la gestión profesional de sus redactores y editores hace que estos nuevos medios sean mucho más que la mera suma de sus partes.

A partir de esta conceptualización de metamedio se puede comprender mejor cómo la curación de contenido es una característica inherente a los metamedios: si se va a trabajar con contenido ya existente de terceros, la curación es fundamental para garantizar la calidad. Antes de repasar la tipología propuesta, cabe recordar brevemente las estrechas asociaciones que hay entre esta práctica y el periodismo que se desarrolla en la Red.

La curación de contenido es una característica inherente a los metamedios: si se va a trabajar con contenido de terceros, la curación es fundamental para garantizar la calidad

La curación o curaduría - del anglosajón content curationha emergido en los últimos años como una necesidad periodística para un escenario marcado por la abundancia de información (McAdams, 2008). El concepto de content curator ha tenido poca competencia en el ámbito del periodismo, entre la que cabría destacar el "periodista DJ" (Rincón, 2013). Éste emerge como el protagonista de un nuevo periodismo basado en la agregación, las relaciones, los relatos transmedia (Scolari, 2013) y las conexiones, donde la experiencia y el sentido crítico del periodista se pone en valor frente a la curación de contenido realizada exclusivamente por algoritmos:

"Aunque lo haga un algoritmo como Google (...) sería mejor que lo hiciera un periodista que ya sabe lo que es poner a diversas voces a narrar en colectivo"; en suma, "el periodista-curador es el que sigue las conversaciones de la comunidad audiencia y que, sobre lo que recoge, crea una narración sublime: vincula lo existente con una macrohistoria" (Rincón, 2013, p. 19).

Guerrini (2013) realiza una aportación explícita a las bases de la curación periodística y a la construcción de un marco conceptual donde empieza a considerarse la curación no sólo como una fase más dentro del ejercicio de la profesión, sino incluso como una redefinición del periodismo en sí mismo tal y como lo conocíamos. En cualquier caso y lógicamente, se trata de una forma de narrativa periodística más propicia para determinadas situaciones y temas, como aquellas que 
generan grandes picos repentinos de conversación social en una comunidad y numerosas dosis aportadas por la participación de la audiencia. Como recuerda Guerrini:

“Algunas historias permiten ser mejor 'curadas' que otras. Las más acertadas son aquellas en las que más participan los usuarios en el proceso de la narración, y más contribuyen con contenido multimedia" (2013, p. 9).

\section{La curación periodística también es un proceso que ayuda al periodismo a re- cuperar su rol de canalizador del espacio público}

Bradshaw (2013) señala por su parte que, si bien el término es relativamente nuevo en el campo periodístico, el periodismo y cada proceso que lo define es un acto de curación en sí mismo. $Y$ aunque algunas historias son más propicias que otras para la curación, esta práctica se convierte en indispensable en un escenario donde la participación y el contenido generado por el usuario están en constante circulación. En este sentido cobra especial importancia subrayar el uso premeditado del concepto de circulación que, frente a la distribución, tiene un carácter más social, interactivo y multidireccional.

Bakker (2014) incluye la curación dentro de los nuevos roles para el periodismo junto con otros como la gestión de comunidades online, al igual que más autores empiezan a subrayar la necesidad de extender esta práctica desde el ámbito de la documentación al del periodismo (Guallar, 2014).

En investigaciones más recientes, la curación periodística se ha planteado también como el proceso que ayude al periodismo a recuperar su rol de canalizador del espacio público, en un escenario comunicativo tan efímero y disperso como el actual. La gran oportunidad es considerar que "la curaduría puede servir para reconstruir el espacio público, ahora fragmentado en una miríada de conversaciones sociales. Y esa sí que es una gran tarea para el periodismo del futuro" (Díaz-Arias, 2015, p. 62).

Parafraseando al autor anterior, podríamos añadir que no sólo se trata del periodismo del futuro, sino incluso del futuro del periodismo. La transición de la curación desde la documentación hasta el periodismo es más natural de lo que parece ya que, como recuerdan Guallar y Leiva-Aguilera (2013), las competencias específicas de la curación de contenido son inherentes a las que siempre ha necesitado un periodista para su desempeño profesional y más si cabe cuando el entorno natural de ese desempeño es la Web.

Como bien recuerda Díaz-Arias (2015, p. 67), no estamos ante una disyuntiva que nos obligue a elegir entre curaduría o periodismo, sino ante el reto de encajar de forma natural al primero dentro del segundo:

"El peligro es que se entienda la curaduría como una sustitución de la creación (...). El periodista añade valor y sentido a los contenidos de los usuarios. Pero eso no quiere decir que deje de crear sus propios contenidos".
Esta consideración es vital para diferenciar la curaduría de la mera agregación automática que puede realizar un algoritmo. Muchos medios y plataformas que se están presentando como herramientas de curación necesitan incorporar a sus procesos a periodistas que doten de pleno sentido al contenido seleccionado.

En septiembre de 2015 ante la noticia de que Twitter estaba contratando a periodistas para mejorar sus procesos de curación de noticias, el profesor Jay Rosen advertía de la necesidad de que prevalezca el criterio personal de un periodista frente a cualquier tipo de algoritmo:

"Twitter contratando a periodistas para la curación de lo mejor de los periódicos es el nacimiento de una nueva bestia editorial. ¿Cómo sabemos si esa bestia tiene alma?" (Jay Rosen, en @jayrosen_nyu el 9 de septiembre de 2015).

Con este repaso sobre las estrechas vinculaciones de la curación con la práctica periodística, se pueden sistematizar varios puntos que la curación de contenido comparte con el marco conceptual necesario para estudiar los metamedios:

\footnotetext{
- añadir nuevos valores o dimensiones a contenidos ya creados;

- trabajar bajo criterios personales, no sólo con algoritmos;

- crear nuevos espacios públicos que perduren en el tiempo;

- rescatar conversaciones e identificar nuevas comunidades.
}

Estos cuatro puntos proceden directamente de la síntesis de lo señalado anteriormente sobre las relaciones entre curación y periodismo, a la vez que ayudan a sistematizar las características deseables en cualquier metamedio actual.

\section{El periodismo todavía no se encuentra cómodo con la participación como pro- ducto en sí mismo}

\section{Taxonomía de metamedios periodísticos}

En la siguiente taxonomía (tabla 1), fruto de una observación no participante sobre los nuevos medios emergentes en la Red, se destacan las prácticas ("característica principal") que han dado lugar a diversos tipos de cibermedios, con sus correspondientes ejemplos. El orden de las categorías no es jerárquico, por lo que no es una escala de mayor a menor o viceversa, sino una representación de la diversidad de cibermedios que podemos encontrar en la actualidad.

Respecto a la unidad de medida utilizada para la elaboración de la tabla, como se trata de estudiar el fenómeno de los metamedios emergentes y bajo las definiciones señaladas anteriormente, no podemos ceñirnos únicamente a la unidad de medida del "medio" o de "sección". Se ha tenido en cuenta cualquier tipo de cabecera con contenido propio en la Web (como por ejemplo un blog), más allá de que ese micromedio o cabecera tuviese una relación jerárquica o de subordinación respecto a la imagen de marca de un medio mayor (como ocurre con los blogs corporativos de medios como 233grados o Pissed off readers). 
Tabla 1. Propuesta para una tipología de nuevos metamedios

\begin{tabular}{|c|c|c|c|}
\hline \multicolumn{4}{|c|}{ Tipología de metamedios periodísticos } \\
\hline Criterio & Tipo & Característica principal & Ejemplos \\
\hline \multirow{4}{*}{ Contenido } & Generalista & $\begin{array}{l}\text { Apoyado en medios verticales anteriores para ampliar } \\
\text { oferta de contenidos }\end{array}$ & $\begin{array}{l}\text { Eldiario.es } \\
\text { Five Magazine }\end{array}$ \\
\hline & Especializado & $\begin{array}{l}\text { Apoyado en medios verticales anteriores para reforzar } \\
\text { un contenido específico }\end{array}$ & Matter + Medium \\
\hline & De producto & $\begin{array}{l}\text { Alianzas temporales alrededor de un producto con me- } \\
\text { dios asociados para repartir producción y difusión }\end{array}$ & Acuerdo + El confidencial \\
\hline & Social & $\begin{array}{l}\text { Nuevos medios basados en la curación de contenido } \\
\text { ajeno }\end{array}$ & $\begin{array}{l}\text { Reported.ly } \\
\text { Storyful.com }\end{array}$ \\
\hline \multirow[b]{2}{*}{ Autores } & Comunitario temporal & Red temporal de periodistas y autores con fin específico & Contributoria.com \\
\hline & Comunitario permanente & $\begin{array}{l}\text { Red permanente de periodistas y autores con fines } \\
\text { diversos }\end{array}$ & GlobalVoices.org \\
\hline \multirow[b]{2}{*}{ Relato } & Periférico & Flujo informativo que nace fuera de las redacciones & Filtrala.org \\
\hline & Teaser & $\begin{array}{l}\text { Asociaciones de medios donde los primeros suponen un } \\
\text { making of de los segundos (teaser) }\end{array}$ & $\begin{array}{l}\text { 233grados + lainformacion } \\
\text { Pissed off... + Acuerdo } \\
\text { Blog + El español }\end{array}$ \\
\hline
\end{tabular}

La presente tipología se inicia, al igual que se ha hecho en el marco conceptual, con la acepción más tradicional y literal de metamedios: aquellos cibermedios formados a partir de medios y contenidos preexistentes. Bajo esta premisa, tres criterios han servido para desarrollar las categorías: contenido, autoría y relato. Cabe destacar que muchas de estas categorías no son excluyentes.

\subsection{Metamedios generalistas}

Como definición operativa, entendemos por metamedios generalistas aquellos apoyados en medios verticales anteriores para ampliar la oferta de contenidos. Cibermedios como Eldiario.es o Five magazine pueden ser ejemplos.

Una de las habilidades de los nuevos medios es articular conexiones entre pequeños medios ya existentes para los que el nuevo gran medio ejerce de conector

Eldiario.es es un medio creado en septiembre de 2012 bajo la dirección del periodista Ignacio Escolar y con una fuerte apuesta por los socios en el modelo de negocio y por la economía y la política en la línea editorial. Si bien podríamos clasificar como tradicional este cibermedio en cuanto a su propuesta tecnológica, deconstruir su composición puede darnos varias pistas sobre la fragmentación y conectividad que caracteriza al actual espacio mediático.

Al dividir la cabecera de Eldiario.es podemos llegar a obtener hasta treinta secciones. De esa treintena, un $37 \%$ son micromedios (blogs, cibermedios verticales, medios unipersonales...) que ya existían antes de septiembre de 2012, antes de que se fundase el cibermedio de Escolar. Además, si de esas secciones se eliminasen aquellas que son opinión, el porcentaje se amplía hasta un $45 \%$.

Con este rápido repaso por la estructura del medio, podemos anticipar que una de las habilidades de los nuevos medios puede ser precisamente articular conexiones entre pequeños medios establecidos anteriormente y para los que el nuevo gran medio ejerce de conector y de esqueleto institucional, económico y periodístico. Al igual que el valor de redes como Twitter o Facebook es su componente holístico como agregador de contenido ajeno, los nuevos medios pueden agregar el contenido y las dinámicas de aquellos cibermedios verticales ya existentes y alineados con su propuesta de valor. De hecho, para algunos medios esa agregación será precisamente su propuesta de valor.

Por su parte Five magazine es el resultado de medios como Yorokobu, Diario Kafka, Politikon, Naukas y Jot Down. Asociaciones de este tipo son cada vez más comunes y respon-

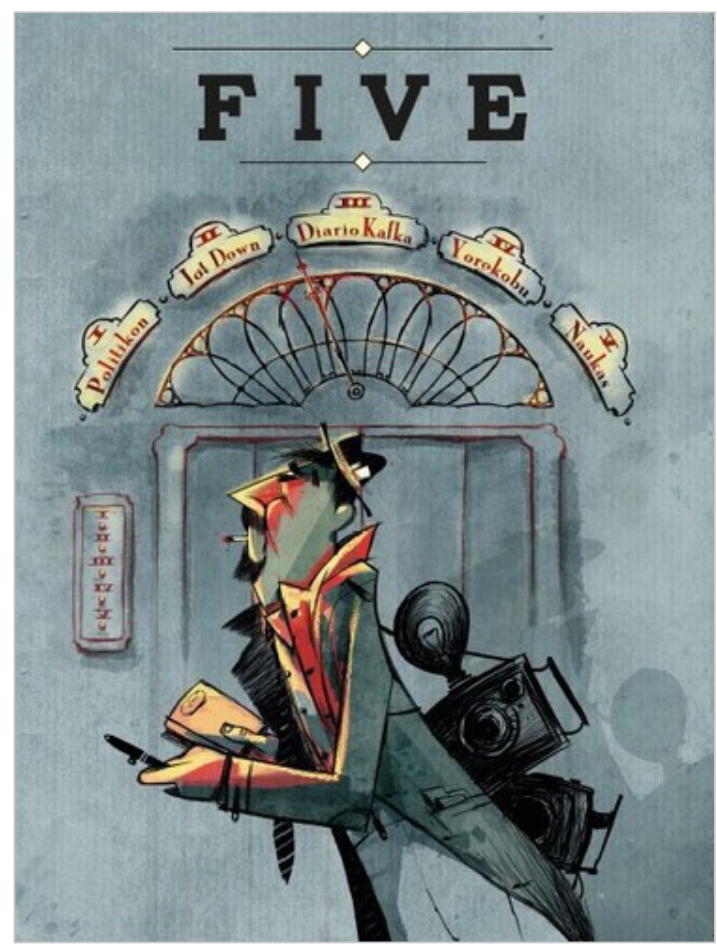

http://www.jotdown.es/store/\#!/ /product/category=2470174\&id=19541216 
den al escenario que estamos describiendo. Las combinaciones pueden ser interminables. Los contenidos de un medio de papel pueden servir de base para los de un medio digital, y los de un cibermedio pueden ser seleccionados $y$ curados para publicarse en papel con otro enfoque. La clave es que estas asociaciones, en la medida en que constituyan asociaciones de calidad, forman medios radicalmente distintos a la mera suma de sus partes. Es de nuevo un recordatorio del carácter holístico que define a un metamedio.

\subsection{Metamedios especializa- dos}

Entendemos por metamedios especializados los que se apoyan en medios verticales anteriores para reforzar un contenido específico.

La asociación entre Matter y Medium es un ejemplo de este tipo de alianzas. El servicio de publicación Medium, fundado por Evan Williams y Biz Stone en 2012, adquirió Matter en abril de 2013, un pequeño cibermedio de información científica que nació a partir de una campaña de crowdfunding y que poco más tarde de su unión con Medium, a principios de 2014, acordó una colaboración con la web española
Materia, dirigida por Patricia Fernández de Lis. Con dicho acuerdo se comprometían a traducir y ofrecer las mejores publicaciones de cada uno a los lectores del otro medio.

Para completar la historia, meses más tarde en septiembre de 2014, Materia se incorpora a Elpais.com para convertirse en su sección de Ciencia. Pocas secuencias como ésta explican tan bien el escenario actual de medios y cómo la conectividad y la colaboración con otros medios ha pasado a ser mucho más importante que el tamaño de las redacciones.

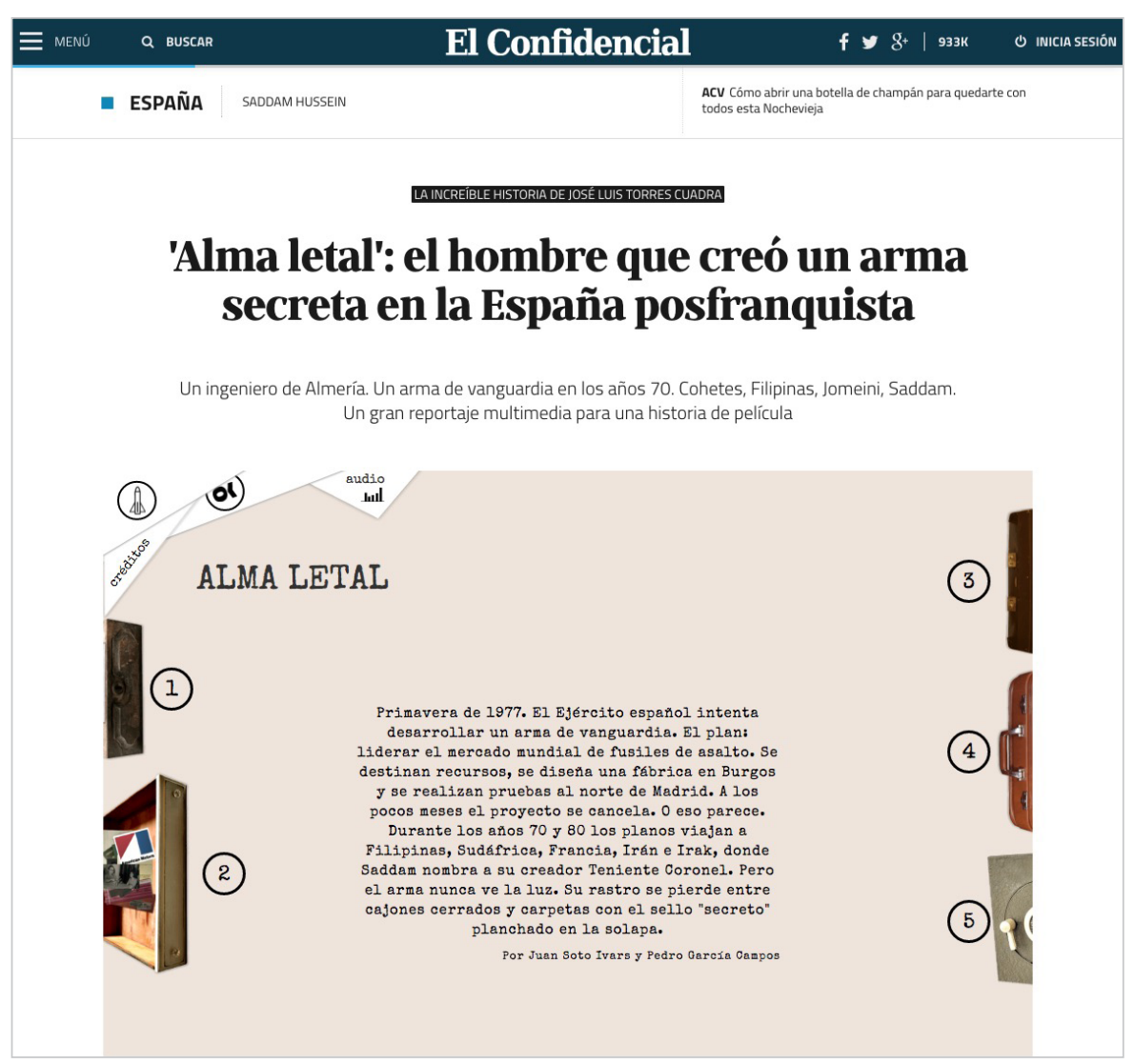

http://www.elconfidencial.com/espana/2014-09-07/el-arma-secreta-de-la-transicion_185309
A su vez, al margen de la asociación con Medium, esta unión de Materia con El País convierte la secuencia en un ejemplo de cómo se forman los metamedios generalistas descritos en la categoría 1. Como se ha señalado al inicio, muchas de estas categorías no son excluyentes; de hecho suelen formar parte de procesos que dan lugar a estructuras informativas nuevas.

\subsection{Metamedios de producto}

Son aquellas alianzas temporales alrededor de un producto, entre medios que se asocian con el fin de repartirse las tareas de producción y difusión. Un caso de este tipo lo encontramos con Acuerdo y Elconfidencial.com, cuando cooperaron con motivo del documental multimedia titulado Alma letal: El hombre que creó un arma secreta en la España posfranquista, publicado por El confidencial el 7 de septiembre de 2014 .

Dicho reportaje fue elaborado por Acuerdo.us, que se asoció con 
este medio para publicar la pieza. ¿Qué ofrecía cada medio? Uno, el know how para hacer una historia concreta; el otro, alcance y temporalidad, una sólida comunidad de usuarios fieles que ayudarán a que el contenido circule y se mantenga vivo por un período mucho mayor que si se hubiese publicado en Acuerdo. http://www.elconfidencial.com/ espana/2014-09-07/el-armasecreta-de-la-transicion_185309

\subsection{Metamedios sociales}

Denominamos así a una nueva generación de cibermedios cuyo rasgo principal es que se fundamentan en la curación de contenido de terceros. Medios como Reported.ly y Storyful. com, o secciones como Verne (EI país) o Enredados (El mundo) representan este tipo de periodismo basado en la verificación y curación de contenido ajeno.

Los metamedios especializados se apoyan en medios verticales anteriores para reforzar un contenido específico

Los dos primeros se distinguen por desarrollar contenido basado en investigar sobre contenidos virales o que pueden serlo, algo que inevitablemente conlleva una curación de contenido digital constante y de calidad. No en vano en la breve presentación del equipo de Reported.ly hay una alusión explícita a la curación de contenido:

"Somos un equipo internacional de periodistas con literalmente decenas de años de valiosa experiencia combinada como organizadores de comunidades online, narradores y curadores".

https://reported.ly
En el caso de Storyful, si bien conviene aclarar que más que un medio es sobre todo una agencia de verificación de contenido interesante en redes sociales, su actividad propicia el tipo de periodismo con el que pueden formarse estos metamedios basados en contenido de terceros.

http://storyful.com

En el panorama español, son secciones específicas de grandes medios las que se han erigido como especialistas de esta meta-información basada en contar la historia de las historias virales en internet, las razones que explican cómo circula determinada información por la Web con mayor velocidad e intensidad que otras historias aparentemente muy similares. Verne en El país y anteriormente Enredados en El mundo son ejemplos de cabeceras que, especialmente en el caso de la primera, son medios en sí mismos.

http://verne.elpais.com

http://www.elmundo.es/enredados.html

\section{D reported.|Y beta}

home reported.ly now digests archives $\vee$ follow us $\vee \quad Q$

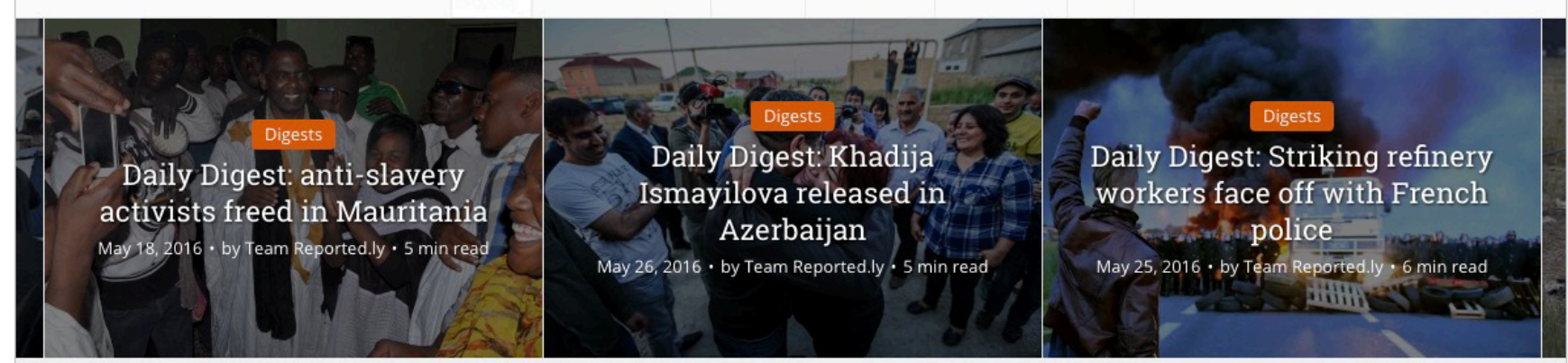

https://reported.ly 


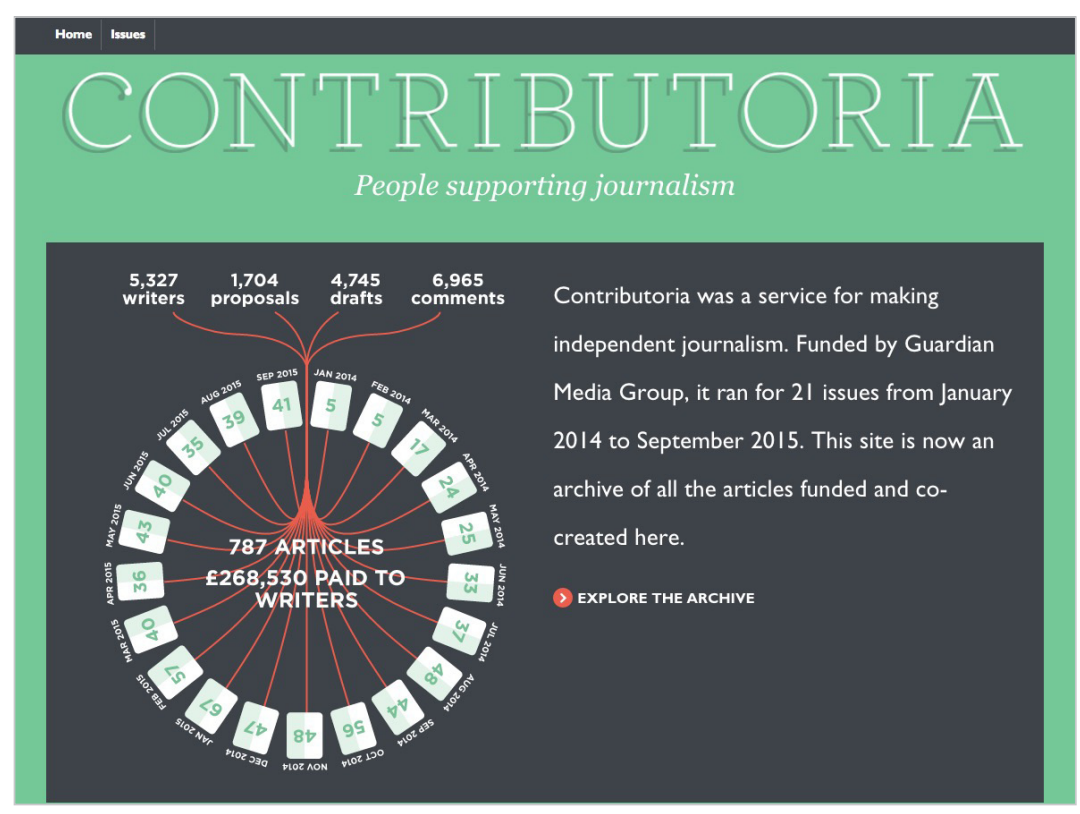

http://www.contributoria.com

\subsection{Metamedios comunitarios temporales}

Denominamos así a los que se forman mediante una red puntual de periodistas y diversos autores con un fin específico. Una actividad que corresponde a esta definición sería Contributoria. Ese carácter líquido y deslocalizado del empleo periodístico en la actualidad, con periodistas individuales que arrastran desde sus perfiles a comunidades mayores que las de algunos medios, también motiva iniciativas temporales articuladas a partir de proyectos específicos, iniciativas que tienen fecha de caducidad.

Contributoria es un ejemplo de este fenómeno y, amparada por el crowdfunding y The Guardian Media Group, esta plataforma conectó a miles de periodistas para que sus historias fueran financiadas y pudieran ver la luz. Entre enero de 2014 y septiembre de 2015, Contributoria aunó a más de 5.000 autores que publicaron bajo este dominio 21 números, con más de 700 artículos. En estos casos hablamos de un periodismo no sólo cofinanciado, sino también coproducido por el alto grado de implicación de los miembros de la comunidad en cada una de las propuestas.

http://www.contributoria.com

\subsection{Metamedios comunita- rios permanentes}

Son aquellas redes que, a diferencia del anterior tipo, se inician con fines diversos y sin fecha de caducidad. El gran ejemplo en esta categoría podría ser uno de los portales históricos de la web colaborativa, Global voices. Traducida a más de 35 idiomas, esta plataforma sin ánimo de lucro recoge más de diez años de historias publicadas sobre más de 160 países. Global voices forma parte ya del imaginario colectivo sobre la corta historia de internet por su papel en la democratización de la información y haber sabido dar voz a minorías multitudinarias. https://globalvoices.org

\subsection{Metamedios periféricos}

Nacen y se organizan desde fuera de las redacciones de los medios convencionales, planteando estructuras y flujos informativos cuyo último eslabón son los medios, como en el caso de Filtrala.org. Muchas de las exclusivas periodísticas que hemos leído en los últimos meses proceden de Fíltrala -con un funcionamiento similar al de Wikileaks- y algunos cibermedios como Crític, Eldiario.es o La marea.

https://filtrala.org

Algunas de las exclusivas destapadas gracias a Fíltrala han sido cómo la empresa Ercros ocultó de forma sistemática que había contaminado con tóxicos un acuífero en Huelva (noviembre de 2015) o que el gobierno estadounidense, en el ámbito del tratado TISA (Trade in Services Agreement), presionó para lograr la liberalización total de catorce servicios profesionales en Europa (diciembre de 2014).

\section{La conectividad y la colaboración con otros medios han pasado a ser mucho más importantes que el tamaño de las redacciones}

Se trata de una plataforma ciudadana donde una persona puede enviar información confidencial a medios de forma anónima y segura. En este caso, como también ocurre con iniciativas como las promovidas por la Fundación Civio, algunas de las mayores innovaciones y disrupciones periodísticas se están produciendo en la periferia del periodismo, desde fuera de las redacciones. La expansión de los medios 


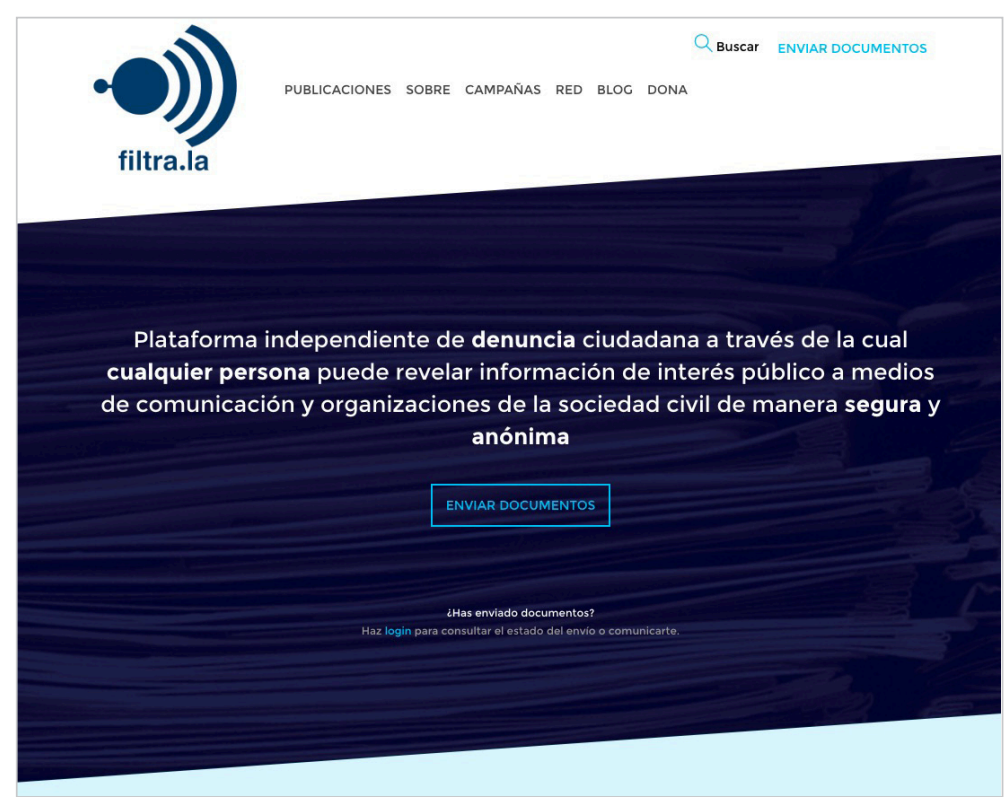

https://filtrala.org

unipersonales, de la marca personal del periodista y de plataformas de publicación, propicia cada vez más este tipo de metamedios, que no son más que asociaciones libres entre fuentes y periodistas.

http://www.civio.es

\subsection{Metamedios teaser}

En último lugar y respecto a la forma de presentar el relato, denominamos metamedios teaser a aquellos utilizados para desarrollar campañas estilo teaser o making of que muchos cibermedios han protagonizado en sus inicios, a menudo utilizando un blog para adelantar el tipo de tono, enfoque y contenidos que iban a predominar en el medio tras su lanzamiento oficial. Podemos reconocer esta estrategia recientemente con El blog de El español, que durante meses publicó y adelantó contenidos antes del lanzamiento oficial de El español, en octubre de 2015. Anteriormente, tenemos ejemplos similares como el blog Pissed off Readers, que ejercía de preludio y making of de Acuerdo.us (a mediados de 2013) y con el blog especializado en periodismo y tecnología 233grados, que sirvió como plataforma para presentar el cibermedio La información, en abril de 2009.

http://www.elespanol.com http://pissedoffreaders.com/es http://233grados.lainformacion. com

\section{Conclusiones}

Al ser una propuesta teórica, el objetivo de este artículo era ofrecer una taxonomía introductoria para la sistematización de los metamedios periodísticos. Esta clasificación, como cualquier herramienta conceptual, está abierta a posteriores mejoras y desarrollos, ya que resulta complicado delimi- tar (en su acepción literal de poner límites) las posibilidades de innovación de un escenario mediático que se encuentra en constante movimiento.

Fruto de ese movimiento hemos podido recopilar numerosos casos en los que se aprovechan medios y contenidos ya existentes para rediseñar nuevos cibermedios o relaciones con fuentes, lectores y periodistas. Relaciones temporales o permanentes, específicas o generalistas, pero todas ellas con algún componente que nos permite pensar bajo la clave de metamedios, un concepto todavía con muy poca bibliografía científica. Podemos validar la hipótesis utilizada como punto de partida en este artículo, ya que todas las nuevas experiencias de ciberperiodismo seleccionadas pueden explicarse bajo algunas de las claves que definen un metamedio.

Los tres criterios que se ofrecen para agrupar hasta ocho categorías de metamedios son los vinculados con el contenido, la autoría y el tipo de relato. Dichas categorías no son excluyentes en muchos de los casos. De hecho, ejemplos como el de Materia nos permitirían hablar incluso de diferentes etapas en las que un metamedio pasa de un estado (sinergias con Medium) a otro (dependencia de El país) experimentando diferentes categorías de las señaladas.

\section{Metamedios sociales son una nueva generación de cibermedios cuyo rasgo principal es que se fundamentan en la curación de contenido de terceros}

Así mismo existen numerosas realidades mediáticas que no aparecen en la tipología presentada pero que no dejan de ser igualmente interesantes y que merecerían por sí mismas ser objeto de estudios más profundos. Por ejemplo, una de esas realidades es el universo selfie que protagonizan perfiles como los youtubers o las it girls de

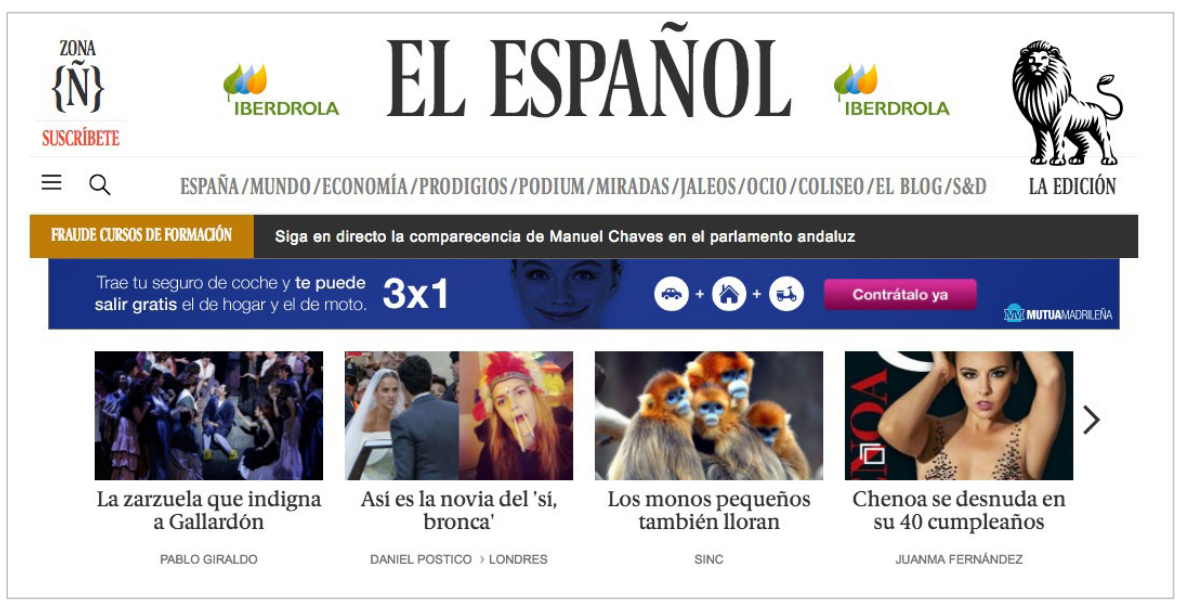

http://www.elespanol.com/ 
Instagram, que llevan a la máxima expresión la idea de micromedio, creando contenidos unipersonales con audiencias de millones de personas en algunos casos. Si bien su carácter de metamedios periodísticos (o incluso de medios) es cuestionable, lo que no cabe duda es que ambos colectivos han creado lenguajes y contenidos nativos para las plataformas desde las que comunican, aprovechando plataformas y redes ya existentes.

Ejemplos como el de Materia permiten hablar de etapas en las que un metamedio pasa de una categoría a otra

\section{Bibliografía}

Bakker, Piet (2014). “Mr. Gates returns: Curation, community management and other new roles for journalists". Journalism studies, v. 15, n. 5, pp. 596-606.

http://dx.doi.org/10.1080/1461670X.2014.901783

Bradshaw, Paul (2013). "Journalism *is* curation: tips on curation tools and techniques". Online journalism blog, 30 Sept.

http://onlinejournalismblog.com/2013/09/30/curationtools-tips-advice-journalism

Campos-Freire, Francisco (2015). "Adaptación de los medios tradicionales a la innovación de los metamedios". El profesional de la información, v. 24, n. 4, pp. 441-450.

http://dx.doi.org/10.3145/epi.2015.jul.11

Díaz-Arias, Rafael (2015). “Curaduría periodística, una forma de reconstruir el espacio público". Estudios sobre el mensaje periodístico, n. esp. diciembre, v. 21, pp. 61-80. http://revistas.ucm.es/index.php/ESMP/article/view/51129

Fidler, Roger (1998). Mediamorfosis. Comprender los nuevos medios. Buenos Aires. ISBN: 9789506412616

Guallar, Javier (2014). “Content curation in journalism (and journalistic documentation)". Hipertext.net, n. 12.

http://raco.cat/index.php/Hipertext/article/view/275781/364536

Guallar, Javier; Leiva-Aguilera, Javier (2013). El content curator. Guía básica para el nuevo profesional de internet. Barcelona: Editorial UOC, colección El profesional de la información, n. 24. ISBN: 9788490640180

Guerrini, Federico (2013). Newsroom curators and independent storytellers: Content curation as a new form of journalism. Oxford: Thomson Reuters Foundation.

https://goo.gl/RN229b
Kay, Alan; Gooldberg, Adele (1977). "Personal dynamic media". Computer, pp. 31-41.

http://www.newmediareader.com/book_samples/nmr-26kay.pdf

http://dx.doi.org/10.1109/C-M.1977.217672

McAdams, Mindy (2008). "Curation, and journalists as curators". Teaching online journalism, 3 Dec.

http://mindymcadams.com/tojou/2008/curation-andjournalists-as-curators

McLuhan, Marshall; Fiore, Quentin (1967). The medium is the message. Nueva York: Bantam Books. ISBN: 978 1584230700

Noguera-Vivo, José-Manuel (2015). Todos todo. Manual de periodismo, participación y tecnología. Barcelona: Editorial UOC. ISBN: 9788491160380

Noguera-Vivo, José-Manuel; Martínez-Sánchez, Jesús-Tomás; Nicolás-Ojeda, Miguel-Ángel; Pérez-Escolar, Marta; Gómez-Company, Ariana; Grandío-Pérez, María-del-Mar; Hernández-Gómez, Francisco; Sánchez-Cobarro, Palomadel-Henar (2014). Economía de la participación. Madrid: Fundación EOI. ISBN: 9788415061458

http://repositorio.ucam.edu/jspui/bitstream/10952/1081/1/ Econom\%c3\%ada_Participacion_EOI_2014.pdf

Rincón, Omar (2013). “El periodista DJ es el medio". En: Luchessi, Lila (coord.). Calidad informativa. Escenarios de postcrisis. Buenos Aires: La Crujía Ediciones, pp. 9-32. ISBN: 978 9876012003

Scolari, Carlos (2013). Narrativas transmedia. Cuando todos los medios cuentan. Bilbao: Deusto. ISBN: 9788423413362

Shirky, Clay (2009). "How social media can make history". TED talks, June.

https://www.ted.com/talks/clay_shirky_how_cellphones_ twitter_facebook_can_make_history/transcript

Toffler, Alvin (1980). The third wave. New York: Bantam Books. ISBN: 9785170624980

Van-Dijk, Jan (2009). "Users like you? Theorizing agency in user-generated content". Media, culture and society, v. 31, n. 1, pp. 41-58.

http://dx.doi.org/10.1177/0163443708098245

Villi, Mikko (2012). "Social curation in audience communities: UDC (user-distributed content) in the networked media ecosystem". Participations. Journal of audience \& reception studies, v. 9, n. 2, pp. 614-632.

http://www.participations.org/Volume\%209/Issue\%20 2/33\%20Villi.pdf

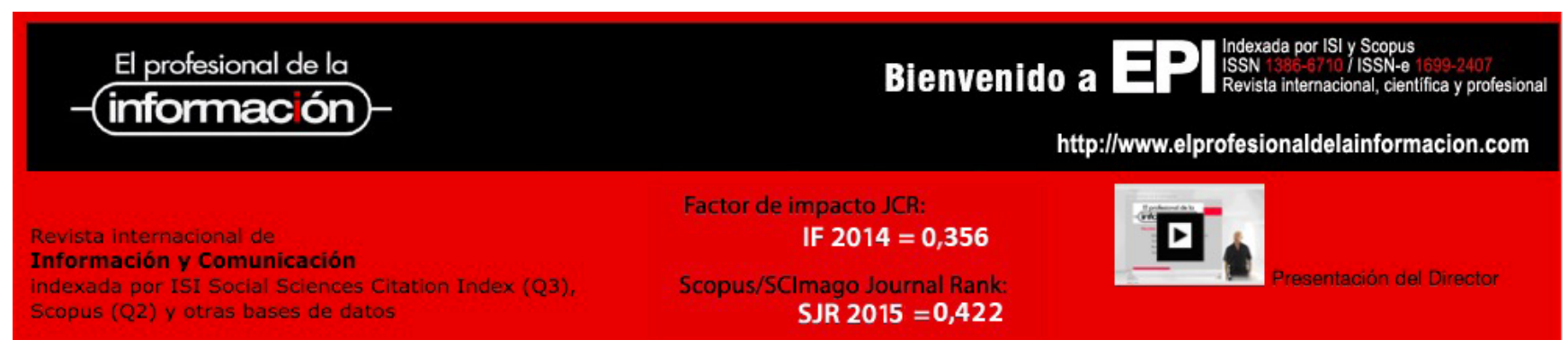

